\title{
Psychometric Evaluation of the Cultural Competence Assessment Instrument Among Healthcare Providers
}

\author{
Ardith Z. Doorenbos $\boldsymbol{\nabla}$ Stephanie Myers Schim $\boldsymbol{\nabla}$ Ramona Benkert $\boldsymbol{\nabla}$ Nagesh N. Borse
}

\section{Editor's Note}

Materials documenting the review process for this article are posted at http://www.nursing-research-editor.com.

Background: The relevance of healthcare provider cultural competency to the achievement of goals for reduction in extant health disparities has been demonstrated; however, there are deficits with regard to cultural competency measurement.

- Objectives: To examine the test-retest reliability of the cultural competence assessment instrument (CCA) among hospice providers, and to examine the reliability and validity of the CCA among healthcare providers in nonhospice settings.

Method: Test-retest reliability of the CCA was assessed using a sample of 51 hospice respondents who completed the CCA at two time points. The internal consistency reliability and construct validity of the CCA for healthcare providers in nonhospice settings were evaluated using a convenience sample of 405 healthcare providers.

- Results: The CCA demonstrated adequate test-retest reliability $(r=.85, p=.002)$ in hospice providers over 4 months. Among healthcare providers in nonhospice settings, the CCA had an internal consistency reliability of .89 overall (.91 and .75 for the two subscales). Construct validity was supported by principal axis factor analysis, which showed two factors with item loadings above .40, explaining $56 \%$ of the variance. Mean scores of the CCA were significantly higher for providers who reported previous diversity training compared to those who had not.

Discussion: Findings for the psychometric properties of the CCA supported its potential as an instrument for measuring provider cultural competence. Knowledge gained will be useful for developing future research studies and specific cultural competence intervention approaches for healthcare providers that may decrease health disparities.

Key Words: cultural competence assessment - healthcare providers · psychometrics
7 he cultural competence of healthcare providers is central to the healthcare system's ability to provide access to and delivery of high-quality, high-value healthcare and it instrumental in reducing health disparities. American society is increasingly diverse in areas such as race, ethnicity, language, religion, gender, sexual orientation, ability, and access to technology. This diversity presents ongoing opportunities for cross-cultural partnerships, but it also creates a multitude of cultural gaps that must be bridged to decrease health disparities (Fortier \& Bishop, 2004). As American society shifts in demographics and attitudes about diversity, there is a growing recognition that health and illness care occurs in dynamic interactions situated within complex cultural contexts for both patients and providers.

The recognition that health and healthcare are not distributed evenly in the United States led to the publication of the 2002 Institute of Medicine (IOM) report Unequal Treatment (Smedley, Stith, \& Nelson, 2003). Since then, the healthcare research community has gained considerable momentum in addressing health disparities and discovering ways to improve healthcare for underserved groups. Yet health disparities continue to exist among many of the racial and ethnic populations in the United States (Long, Chang, Ibrahim, \& Asch, 2004). The U.S. Department of Health and Human Services [DHHS] Centers for Disease Control and Prevention (2004) has

Ardith Z. Doorenbos, PhD, RN, is Postdoctoral Fellow, College of Nursing, Michigan State University, East Lansing.

Stephanie Myers Schim, PhD, RN, is Assistant Professor; and Ramona Benkert, PhD, RN, is Assistant Professor, College of Nursing, Wayne State University, Detroit, Michigan.

Nagesh N. Borse, MS, PGDPM, BPharm, is International Health Doctoral Student, Bloomberg School of Public Health, Johns Hopkins University, Baltimore, Maryland. 
identified large disparities in infant mortality rates among racial and ethnic groups and a persistent gap in life expectancy between Black and White populations. In addition, disparities in risk factors, morbidity, and access to healthcare exist for persons of Hispanic origin. American Indians are more likely to be uninsured than those in other racial and ethnic groups.

Patterns of culturally incompetent care from providers influence these health disparities. Numerous studies have suggested that racial differences influence communication, resulting in inadequate diagnostic testing (Canto et al., 2000), miscommunications about etiologies, insufficient treatment plans (Abreu, 1999), and discounting of patient's ideas (Helms \& Cook, 1999). Appropriate care across cultures can occur only when patient, family, and community expectations are aligned with provider knowledge, attitude, and behavior. Supporting this observation, one of the key recommendations of the IOM report is to integrate crosscultural education into the training of all healthcare professionals (Smedley et al., 2003).

Expansion of culturally congruent care promises to enhance the attainment of most Healthy People 2010 objectives (DHHS, Office of Disease Prevention and Health Promotion [ODPHP], 2001). It is particularly relevant to Objectives 1-6 (reduce the proportion of families that experience difficulty or delay in getting healthcare), 11-6 (increase the proportion of persons who report that their providers have satisfactory communication skills), and 7-11 (increase the number of local health departments with culturally and linguistically competent programs). Increased compatibility between clients and providers as they bridge cultural differences holds the promise of reducing health disparities (DHHS, ODPHP, 2001); the relevance of provider cultural competency in the reduction of racial and ethnic health disparities has been clearly articulated (Ahmann, 2002; Brach \& Fraser, 2000). However, deficits in effective cultural competence measurement remain (Geiger, 2001).

Although no simple formula exists for measuring the complex interplay of sensitivity, knowledge, behaviors, and awareness - all elements of culturally attuned nursingthere is some progress in explicating the essentials of a cultural competence process model (Campinha-Bacote, 2002; Doorenbos \& Schim, 2004). Still, many of the instruments available to measure cultural competence are focused on testing racial or ethnic group-specific knowledge (Bernal \& Froman, 1987; Rooda, 1993) or are adaptations of attitude (Rooda, 1993), knowledge (Rooda, 1993), or selfefficacy scales (Bernal \& Froman, 1987), which only operationalize select aspects of a broader concept of cultural competence.

The three most widely used instruments in this area of nursing research are the Inventory for Assessing the Process of Cultural Competence among Health Care Professionals (IAPCC; Campinha-Bacote, 1999), the Cultural Self-Efficacy Scale (CSES; Bernal \& Froman, 1987), and the Cultural Attitude Survey-Modified (CAS-M; Rooda, 1993).

Despite its demonstrated reliability and validity and linkages to a cultural competence process framework, the IAPCC was written at an advanced reading level and contains five different response sets for the 20-item instrument. The IAPCC requires readers to shift between various re- sponse levels simultaneously, which has proven problematic for interdisciplinary teams and groups with mixed educational levels.

Although the CSES has significant reliability ( $\alpha=$ .97-.98; Bernal \& Froman, 1987; Smith, 1998) and content validity, it is not linked to an overarching cultural competence model. Smith (2001) linked the instrument to the Geiger and Davidhauser framework, but the focused conceptualizations of the instrument to skill and knowledge required the use of an additional instrument (the CAS-M; Rooda, 1993) for the measurement of cultural competence attitudes. The CSES is also quite long (58 items) and is limited to the measurement of participants' confidence with three specific racial and ethnic groups.

The CAS-M (Rooda, 1993) was designed to measure attitudes towards and knowledge about three specific racial and ethnic groups: African American, Hispanic, and Asian American. Although the CAS-M has obtained less than adequate reliability across a variety of studies (Rooda, 1993; Smith, 2001), it continues to fill a void for researchers interested in measuring cultural competence.

A further limitation of the existing instruments is that many were designed for a particular healthcare discipline, with specific knowledge not applicable to healthcare providers across a spectrum of disciplines and educational levels (Benkert, Tanner, Guthrie, Oakley, \& Pohl, in press; Campinha-Bacote, 1999; Jibaja-Rusth, Kingery, Holcomb, Pruitt, \& Buckner, 1994; Motwani, Hodge, \& Crampton, 1995; Sodowsky, Taffe, Gutkin, \& Wise, 1994). The most frequently used instrument to measure broad cultural competence in psychology and other counseling professions is the Multicultural Counseling Inventory (MCI; Sodowsky et al., 1994), which was designed to follow the broad crosscultural counseling competencies developed by Sue et al. (1982). Although the instrument has demonstrated good reliability (.89) and content validity with numerous counseling professionals (Bellini, 2003; Sodowsky et al., 1994), the one instance of its use with junior nursing students did not match these results $(\alpha=.69-.81$; Pope-Davis, Eliason, \& Ottavi, 1994).

As outlined above, measurement instruments that are valid and reliable across the broad range of cultures, disciplines, work roles, and educational levels employed in U.S. healthcare are notably lacking (Fortier \& Bishop, 2004; Schim, Doorenbos, Miller, \& Benkert, 2003). Only the IAPCC and the CCA employed in this study are based on an existing cultural competence framework or model and the IAPCC is unsuited to interdisciplinary research. The CCA presents the possibility of an effective and valid instrument for measuring cultural competence across disciplines and educational levels. Thus, the purpose of this study is (a) to examine the test-retest reliability of the CCA among hospice providers and (b) to examine the reliability and validity of the CCA among healthcare providers in nonhospice settings. Evaluating the psychometric properties in healthcare providers is necessary to support the use of the CCA in determining cultural competence progress and outcomes. The CCA can then be used as a research instrument for evaluating the effectiveness of interventions designed to increase cultural competence based on the cultural competence model. 


\section{Conceptual Model}

The cultural competence model has been described using the analogy of a jigsaw puzzle, where pieces represent important provider elements (Figure 1). The model, which has four elements (cultural diversity, cultural awareness, cultural sensitivity, and cultural competence), was the foundation for development of the CCA (Doorenbos \& Schim, 2004). Culture, as classically defined in anthropology by Tylor (1958), is "that complex whole which includes knowledge, belief, arts, morals, law, custom, and many other capabilities and habits acquired by man as a member of society" (p. 1). This definition is focused on attributes acquired through living in or experiencing a particular society, rather than through biological inheritance (Kottak, 2004). Leininger (1991) further defines culture as "the learned and transmitted values, beliefs, and practices that provided a critical means to establish culture-care patterns from the people” (p. 36). The fusion of these definitions leads to an understanding of $\mathrm{cul}$ tural competence as the demonstration of knowledge, attitudes, and behaviors based on diverse and relevant cultural experiences. It is not expected that healthcare providers achieve complete cultural competence, but rather that they continue to strive to match their competencies to the specific populations and subgroups with whom they work.

Cultural diversity is increasingly recognized as a complex and dynamic reality of modern American healthcare. Diversity is a fact of life in healthcare settings among both provider and patient populations. Individual experiences with a diversity of people and groups vary in depth and scope. Provider exposures and experiences widely vary with regard to cultural realities and the number and types of people encountered.

Cultural awareness relates to provider knowledge about those areas of cultural expression in which groups tend to differ and those in which similarities are noted. Much of the existing work in cultural competence development is focused on descriptions of major differences in such areas as language, kinship patterns, religion, and food

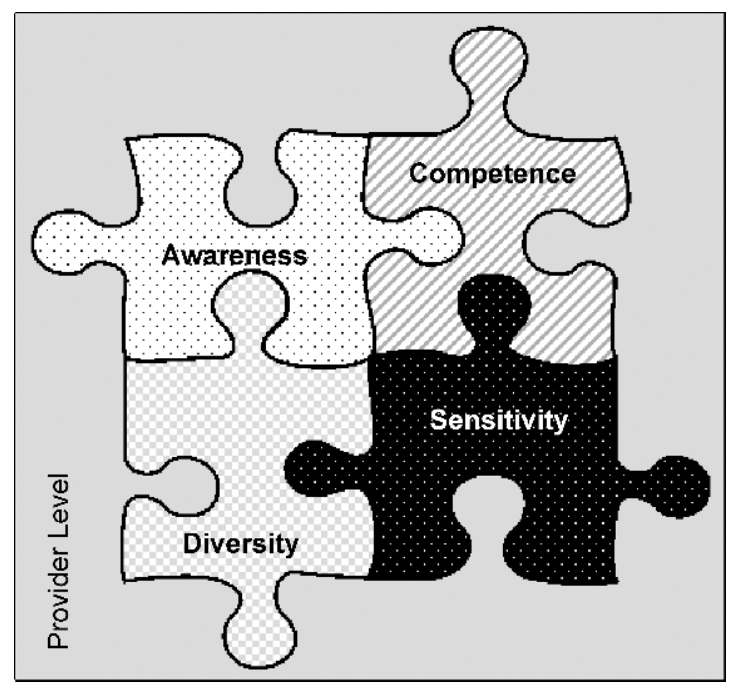

FIGURE 1. Cultural competence model. that are observed across diverse groups. Effective cultural awareness, however, needs to be based on knowledge of ways in which individual differences and similarities traverse cultural group boundaries, so that providers can appropriately assess individuals, families, and communities within their cultural contexts.

Cultural sensitivity describes provider attitudes, values, beliefs, and personal insight. Acknowledgement of personal heritage and beliefs, openness to "otherness," and respect for the complex ways in which cultural issues influence every aspect of healthcare are central to being a culturally sensitive healthcare provider.

Cultural competence behaviors are the observable outcomes of diversity experience, increased awareness, and refinement of sensitivity. Appreciation, adaptation, negotiation, and explication of healthcare that is culturally congruent with patient needs and wants more often result in effective and efficient healthcare interactions (Fortier \& Bishop, 2004). Consistent demonstration of behaviors such as focusing cultural assessment, asking about explanatory models and expectations for care, adapting interventions to respect cultural practices or taboos, and seeking additional information and resources are hallmarks of cultural competence. To expand the scope and depth of overall cultural competence, all four elements must be developed: (a) cultural diversity experiences, (b) extension of cultural awareness (knowledge), (c) adoption of culturally sensitive attitudes, and (d) actual demonstration of culturally competent behaviors.

The cultural competence model rests upon a broad definition of culture and cultural diversity, allowing for application across a broad range of cultural realities. This breadth of application, as well as the focus on increasing knowledge and awareness of cultural concepts, the inclusion of the cultural sensitivity dimension, and the goal of developing cultural competence behaviors, fits well with recent national recommendations (DHHS, Health Resources and Services Administration, 2001). The model has proven useful also in framing the development of the evolving instrument for measurement of cultural competence among healthcare providers.

\section{Previous Instrument Development and Testing}

The first version of the CCA consisted of 45 items developed using the tailored design method (Dillman, 1999). One index and three subscales operationally defined the four pieces of the puzzle at the provider level: diversity experience, cultural awareness, cultural sensitivity, and cultural competence behaviors. The CCA then underwent two rounds of expert review to establish content and face validity, and a face-to-face field test to evaluate the cognitive and motivational factors that may influence response choices. The version of the CCA used for the initial reliability and validity evaluation with the hospice sample consisted of 38 scaled items remaining after the expert review and face-to-face field test.

Initial reliability and validity testing was conducted with a group of 113 hospice providers. Internal consistency reliability for the CCA was .92. Internal consistency for the Cultural Competence Behaviors (CCB) subscale was .93 and for the Cultural Awareness and Sensitivity (CAS) 
was .75 . Construct validity was established by factor analysis. Further validity was established by significant moderate correlation of CCA scores to an instrument with similar underlying constructs, the IAPCC (Campinha-Bacote, 1999). Additionally, the CCA scores among hospice providers reporting previous diversity training were significantly different from those having no diversity training, indicating that the CCA is sensitive to differences in contrasted groups (Schim et al., 2003). Thus, CCA performed well as a measure of cultural competence among hospice providers representing a wide range of educational levels and backgrounds.

\section{Methods}

Prior to contacting potential participants for either study, approval was obtained from the appropriate university and healthcare systems human subject investigation committees.

\section{Test-Retest Reliability}

The interval of time between administrations of the CCA may be an important variable in limiting its reliability. In a study to examine the effectiveness of a cultural competence educational intervention, the CCA was used in a quasi-experimental crossover design with hospice providers representing a variety of disciplines, job roles, and education levels. Hospice providers completed the CCA before and after both cultural competence (intervention) and ethics (control) educational sessions. The crossover design of this study allowed for the measurement of the test-retest reliability using Pearson's product-moment correlation analysis among the 51 hospice providers in the control condition over 4 months.

\section{Reliability and Construct Validity in Healthcare Providers}

A convenience sample of 405 healthcare providers was recruited from seven hospitals, a community health agency, and a home health agency. Each potential participant was approached while off-duty, before or after a work shift, using face-to-face contact. A research assistant familiar with each of the hospitals and agencies asked that providers complete the pencil-and-paper survey and return it in an unmarked envelope. Participants were asked not to put names or other identifying marks on the survey forms to ensure anonymity and were advised that participation was completely voluntary. The survey took 20-30 minutes to complete. Return of the survey constituted informed consent.

Internal consistency of the CCA in healthcare providers was evaluated by determining the Cronbach's alpha coefficients for the overall scale and subscales. An unpaired $t$ test (two-tailed) was used to investigate any differences between the CCA scores of providers who reported having diversity training and those who had not.

This study used the same initial 38 items and factor analytic approach that were used when the hospice provider sample was analyzed: principal axis factoring using the oblimin (nonorthogonal) rotation method with Kaiser normalization. An a priori criterion of .40 was used for inclusion of items in factors. Standard sample size esti- mates for adequate factor analysis suggest using at least 10 times more observations than items (MacCallum, Widaman, Zhang, \& Hong, 1999). In this study, the achieved sample size was 405 for 38 items, indicating that the sample size was adequate for factor analysis $(>10: 1$ ratio). Additionally, the Kaiser-Meyer-Olkin measure of sampling adequacy was .86 , which exceeds the .60 that is recommended (Kaiser, 1974).

\section{Instruments}

The CCA is designed to measure cultural diversity experience, cultural awareness and sensitivity, and cultural competence behaviors. Cultural diversity experience is a single-item index asking respondents to identify whether they have encountered people of various groups in the past 12 months. The item score is a simple count of the number of groups endorsed, a higher number indicating greater diversity of experience.

The CAS subscale measuring cultural awareness and sensitivity is measured with a 5-point Likert-like response set of strongly agree, agree, disagree, strongly disagree, and no opinion. The CCB subscale measuring cultural competence behaviors has response categories of always, often, at times, never, and not sure. Summing items from the CAS and CCB subscales yields the subscale scores. Higher scores indicate higher levels of knowledge, more positive attitudes, and greater frequency of competence behaviors. Internal consistency reliability for the CCA has been reported at .92; Cronbach's alphas for the CCB and CAS subscales were reported at .93 and .75 , respectively; and content and face validity have been established for all items (Schim et al., 2003).

Demographic questions include age, education, and selfidentified ethnic-racial category. Gender is not assessed, due to the possibility of positively identifying individuals in disciplines with a low prevalence of certain gender groups. Years of healthcare experience were assessed by asking providers to write how many years they had been practicing healthcare providers. Prior diversity training was assessed using a yes or no item.

\section{Results}

\section{Test-Retest Reliability}

Respondent Characteristics The 51 hospice providers in the crossover design control group (those that received the ethics program first) were used for the CCA test-retest reliability. Demographic characteristics of the hospice provider sample are presented in Table 1.

Test-Retest Reliability Test-retest reliability for the overall scale showed high correlation $(r=.85, p=.002)$. The CCB $(r=.87, p=.002)$ and CAS subscales $(r=.82, p=$ .002 ) of the CCA also demonstrated high correlations over the 4-month period.

\section{Reliability and Construct Validity in Healthcare Providers}

Healthcare Provider Characteristics The demographic characteristics for the healthcare provider sample are shown in Table 1. 
TABLE I. Demographic Characteristics of Samples

\begin{tabular}{lcc} 
& $\begin{array}{c}\text { Hospice } \\
\text { Workers } \\
(\boldsymbol{n}=\mathbf{5 1})\end{array}$ & $\begin{array}{c}\text { Healthcare } \\
\text { Providers } \\
(\boldsymbol{n}=405)\end{array}$ \\
\hline Age (in years), mean $[S D]$ & $46[11.4]$ & $41[11.7]$ \\
Highest educational level & & \\
Less than high school & $1(2 \%)$ & $4(1 \%)$ \\
High school diploma & $13(25 \%)$ & $62(15 \%)$ \\
Some college & $10(19 \%)$ & $75(19 \%)$ \\
College degree & $11(20 \%)$ & $106(26 \%)$ \\
Graduate school & $16(31 \%)$ & $146(36 \%)$ \\
Racial/ethnic self-identification & & \\
White & $40(78 \%)$ & $262(65 \%)$ \\
Black & $4(14 \%)$ & $76(19 \%)$ \\
Native American & $1(2 \%)$ & $14(4 \%)$ \\
Hispanic & $1(2 \%)$ & $7(2 \%)$ \\
Other & $2(4 \%)$ & $46(11 \%)$ \\
Provider discipline & & \\
Nursing (RN/LPN) & $14(27 \%)$ & $165(41 \%)$ \\
Nursing assistant & $11(22 \%)$ & $46(11 \%)$ \\
Social work & $6(11 \%)$ & $59(15 \%)$ \\
Clergy & $4(7 \%)$ & - \\
Other (clerical, administrative, \\
therapist, etc.) & $16(33 \%)$ & $135(33 \%)$ \\
& & \\
& &
\end{tabular}

Reliability The Cronbach's alpha of the CCA for healthcare providers $(n=405)$ was .89 , using items of the CAS and CCB subscales. The Cronbach's alpha was .91 for the 16-item CCB and .75 for the 11-item CAS, indicating acceptable reliability for the two subscales. This suggests that the CCA and the two subscales exhibited a high level of internal consistency reliability for this sample. The Cronbach's alpha-if-item-deleted ranged from .89 to .87 , indicating that no items were unreliable. Correlation coefficients ranging from .32 to .60 were calculated for the corrected item-total correlations; no items indicated that they should not be a part of the scale (below .30). According to the interitem correlation matrix, none of the items were above .80 , indicating a lack of multicollinearity (Tabachnick \& Fidell, 2001).

Factor Analysis Principal axis factoring using the oblimin rotation method was used to investigate the CCA factors among healthcare providers on the basis of the cultural competence model. The factor analysis included items representing the awareness, sensitivity, and behavior components of the cultural competence model, but did not include the cultural diversity item.

Selection of the number of CCA factors among healthcare providers was based on eigenvalues, scree test, factor correlation matrix, and well-defined loadings of over .40. Six factors had eigenvalues greater than one; thus, the screen test of eigenvalue plots was examined for discontinuity of values. The first factor had an eigenvalue of 6.9 and the second factor 3.8, whereas the next four values were 1.8 , $1.3,1.2$, and 1 , revealing a discontinuity of values between the second and third eigenvalues. The six-factor correlation matrix was examined to see how the factor solution related to the correlation matrix. The correlation matrix showed moderate correlation between Factors 1 and 2. Correlations between Factor 1 and Factors 3-6 were weak or negative in direction (Table 2). In the six-factor solution, Factor 3 had two items loading over .40, however, one item was poorly defined and loaded onto another factor, leaving a single-item clearly defining this factor. Factors 4, 5 , and 6 had no items loading over .40 .

Based on the discontinuity of eigenvalues between Factors 2 and 3, having only weak or negative correlations between Factor 1 and Factors 3-6, and having either poorly differentiated factor loadings or factor loadings that were not above 40 in Factors 3-6, a two-factor solution was determined to best fit the healthcare provider data (Table 3). Sixteen behavior items loaded onto the first factor and accounted for $38 \%$ of the total variance. The second factor was composed of 11 cultural awareness and sensitivity items and accounted for $18 \%$ of the total variance.

Contrasted-Groups Assessment The ability to determine cultural competence differences in healthcare providers using the CCA was assessed using the item which ascertained whether the healthcare provider had previous diversity training. It was hypothesized that compared with the group with no previous diversity training, CCA scores for those having previous diversity training would be higher. As hypothesized, scores for providers who reported receiving previous diversity training were significantly higher than those who reported no training, $t(392)=$ $2.22, p<.001$, two-tailed. This finding suggests sensitivity of the CCA in detecting differences in cultural competence among healthcare providers.

\section{Discussion}

Lack of standardized evaluative measures has been cited as one of the major methodological challenges in cultural

TABLE 2. Factor Correlation Matrix for

Six-Factor Solution

\begin{tabular}{lcccccc} 
& $\begin{array}{c}\text { Factor } \\
\mathbf{1}\end{array}$ & $\begin{array}{c}\text { Factor } \\
\mathbf{2}\end{array}$ & $\begin{array}{c}\text { Factor } \\
\mathbf{3}\end{array}$ & $\begin{array}{c}\text { Factor } \\
\mathbf{4}\end{array}$ & $\begin{array}{c}\text { Factor } \\
\mathbf{5}\end{array}$ & $\begin{array}{c}\text { Factor } \\
\mathbf{6}\end{array}$ \\
\hline Factor 1 & 1.00 & & & & & \\
Factor 2 & .347 & 1.00 & & & & \\
Factor 3 & .093 & .134 & 1.00 & & & \\
Factor 4 & .116 & .120 & .053 & 1.00 & & \\
Factor 5 & -.030 & .002 & .336 & .281 & 1.00 & \\
Factor 6 & -.322 & -.053 & .067 & -.212 & -.274 & 1.00
\end{tabular}


TABLE 3. Factor Solution of the Awareness, Sensitivity, and Behavior Items $(n=405)$

\begin{tabular}{|c|c|c|}
\hline & Communalities & Factor Loadings \\
\hline Factor 1: Behavior & Eigenvalue: 6.91 & $\%$ of variance: $38 \%$ \\
\hline I include cultural assessment when I do client or family evaluations. & .489 & .616 \\
\hline I seek information on cultural needs when I identify new clients and families in my practice. & .562 & .666 \\
\hline $\begin{array}{l}\text { I have resource books and other materials available to help me learn about clients and families } \\
\text { from different cultures. }\end{array}$ & .448 & .565 \\
\hline I use a variety of sources to learn about the cultural heritage of other people. & .532 & .653 \\
\hline I ask clients and families to tell me about their own explanations of health and illness. & .500 & .622 \\
\hline I ask clients and families to tell me about their expectations for care. & .530 & .651 \\
\hline I avoid using generalizations to stereotype groups of people. & .301 & .491 \\
\hline I recognize potential barriers to service that might be encountered by different people. & .372 & .557 \\
\hline I act to remove obstacles for people of different cultures when I identify such obstacles. & .554 & .645 \\
\hline $\begin{array}{l}\text { I act to remove obstacles for people of different cultures when clients and families identify such } \\
\text { obstacles to me. }\end{array}$ & .574 & .688 \\
\hline I welcome feedback from clients about how I relate to others with different cultures. & .720 & .669 \\
\hline I welcome feedback from co-workers about how I relate to others with different cultures. & .723 & .650 \\
\hline I find ways to adapt my services to client and family cultural preferences. & .533 & .680 \\
\hline I document cultural assessments. & .524 & .649 \\
\hline I document the adaptations I make with clients and families. & .538 & .650 \\
\hline I learn from my co-workers about people with different cultural heritages. & .251 & .472 \\
\hline Factor 2: Awareness and Sensitivity & Eigenvalue: 3.8 & $\%$ variance: $18 \%$ \\
\hline Race is the most important factor in determining a person's culture.* & .310 & .539 \\
\hline People with a common cultural background think and act alike.* & .288 & .454 \\
\hline Many aspects of culture influence health and healthcare. & .325 & .518 \\
\hline Aspects of cultural diversity need to be assessed for each individual, group, and organization. & .311 & .531 \\
\hline $\begin{array}{l}\text { If I know about a person's culture, I do not need to assess their personal preferences for } \\
\text { health services.* }\end{array}$ & .488 & .667 \\
\hline Spirituality and religious beliefs are important aspects of many cultural groups. & .316 & .503 \\
\hline Individuals may identify with more than one cultural group. & .266 & .535 \\
\hline Language barriers are the only difficulties for recent immigrants to the United States.* & .452 & .606 \\
\hline $\begin{array}{l}\text { I understand that people from different cultures may define the concept of "healthcare" in } \\
\text { different ways. }\end{array}$ & .365 & .567 \\
\hline $\begin{array}{l}\text { I think that knowing about different cultural groups helps direct my work with individuals, families, } \\
\text { groups, and organizations. }\end{array}$ & .382 & .663 \\
\hline I enjoy working with people who are culturally different from me. & .408 & .404 \\
\hline
\end{tabular}

*Reverse-scored items.

competence research (Fortier \& Bishop, 2004). Without valid and reliable measurement instruments, it is not possible to link cultural competence interventions for healthcare providers to improvements in health outcomes. The CCA was developed to address this gap, and the results to date are encouraging. The CCA has demonstrated satisfactory internal consistency reliability, indicating that CCA items are reliable. The result of the significant correlation found between the administrations of the CCA suggests that cultural competence is a relatively stable construct. The stability of the CCA and both subscales (CAS and CCB) provides evidence of satisfactory test-retest reliability. The 4-month time interval between retests suggests that the CCA and its subscales are not susceptible to the influence of extraneous factors from one administration to the next, and the retest measures are statistically independent (McDonald, 1999).

A contrasted-groups analysis was used for testing the sensitivity of the CCA for detecting differences in cultural competence levels between two groups: those with previous 
diversity training and those who reported no training. The significantly higher scores on the CCA in providers with previous diversity training suggest that educational interventions providing cultural competence training influence CCA scores.

Among healthcare providers, a two-factor solution was found to best fit the data. This finding may suggest that healthcare providers consider knowledge and attitudes as inseparable cognitive components of cultural competence. Cultural competence behavior was a clear and strong factor in healthcare providers, as indicated by the consistently strong factor loadings for items of the CCB subscale. These findings lend support to the reliability and validity of the CCA. As the CCA continues to be refined, ongoing psychometric evaluation is indicated.

There are certain limitations in this study. First, the data were obtained from convenience samples of healthcare providers rather than from randomly selected subjects; therefore, findings must be interpreted with caution. The subscales of the CCA are considered preliminary, pending further validation. Future research should include instrument testing using a stratified random sample. Additionally, the CCA relies on self-report of all aspects of cultural competence rather than on directly observed knowledge, attitude, and behaviors. In light of the potential for respondents to be heavily influenced by social desirability, subsequent versions of the CCA have included the Marlowe-Crowne scale, which assesses social desirability, as an additional evaluation tool (Reynolds, 1982).

Cultural diversity experience is theoretically linked to awareness, sensitivity, and competence behaviors. The use of the simple cultural diversity index to assess respondents' experiences with various racial and ethnic groups does not sufficiently capture either the breadth or the depth of individual experiences with diverse populations. A more effective measure of this piece of the puzzle is needed. In subsequent versions of the CCA, additional categories have been added to more closely reflect the broad definition of culture being used. For example, responses for diverse populations such as persons with disabilities; persons in the gay, lesbian, bisexual, and transgendered community; and persons of different religious affiliations are presented as options. Additionally, the cultural diversity experience items are scaled for the amount of contact with each group. This revision will allow for diversity experience to be treated as a subscale comparable to the CAS and $\mathrm{CCB}$, to be included in the overall CCA scale, and to be included in ongoing psychometric evaluation.

To decrease health disparities in healthcare settings, there is a need to increase cultural competence among providers; however, the difficulty of how to measure provider cultural competence presents an obstacle to this endeavor. The CCA has demonstrated good reliability and adequate construct validity, supported by factor analysis. In light of these promising aspects of the results, there is potential utility in using the two subscales of behavior (CCB) and awareness and sensitivity (CAS), as well as the overall cultural competence (CCA), to assess cultural competence among healthcare providers with diverse disciplinary, educational, and ethnic and racial backgrounds, working in a variety of healthcare settings.
Accepted for publication April 16, 2005.

Research Support: Sigma Theta Tau: Kappa Iota Chapter Assessment of Cultural Competence in Hospice: PI Ardith Doorenbos; American Nurses Foundation grant \#2002047 Enhancing Cultural Competence in Hospice: PI Stephanie Schim.

The authors thank Karyn Huenemann, MPhil, Simon Fraser University, for her editing of the manuscript, and Cathy F. Gretch, Debra A. Amaro, Lelita D. Doctora, and Nancy A. Higgins, Masters candidates at Wayne State University, College of Nursing, for their assistance with data collection.

Corresponding author: Stephanie Myers Schim, PhD, RN, College of Nursing, Wayne State University, 240 Cohn Building, Detroit, MI 48202 (e-mail:s.schim@wayne.edu).

\section{References}

Abreu, J. M. (1999). Conscious and unconscious African American stereotypes: Impact on first impression and diagnostic ratings by therapists. Journal of Consulting and Clinical Psychology, 67, 387-393.

Ahmann, E. (2002). Developing cultural competence in health care settings. Pediatric Nursing, 28, 133-137.

Bellini, J. (2003). Counselors' multicultural competencies and vocational rehabilitation outcomes in the context of counselor-client racial similarity and difference. Rehabilitation Counseling Bulletin, 46, 164-173.

Benkert, R., Tanner, C., Guthrie, B., Oakley, D., \& Pohl, J. (2005). Cultural competence in nurse practitioner students: A consortium's experience. Journal of Nursing Education, 44(5), 225-233.

Bernal, H., \& Froman, R. (1987). The confidence of community health nurses in caring for ethnically diverse populations. IMAGE: The Journal of Nursing Scholarship, 19, 201-203.

Brach, C., \& Fraser, I. (2000). Can cultural competency reduce racial and ethnic health disparities? A review and conceptual model. Medical Care Research and Review, 57(Suppl. 1), 181-217.

Campinha-Bacote, J. (1999). A model and instrument for addressing cultural competence in health care. Journal of Nursing Education, 38, 203-207.

Campinha-Bacote, J. (2002). The process of cultural competence in the delivery of healthcare services: A culturally competent model of care (4th ed.). Cincinnati, $\mathrm{OH}$ : Transcultural C.A.R.E. Associates.

Canto, J. G., Allison, J. J., Kiefe, C. I., Fincher, C., Farmer, R., Sekar, P., et al. (2000). Relation of race and sex to the use of reperfusion therapy in Medicare beneficiaries with acute myocardial infarction. New England Journal of Medicine, 342, 1094-1100.

Dillman, D. A. (1999). Mail and Internet surveys: The tailored design method (2nd ed.). New York: Wiley.

Doorenbos, A. Z., \& Schim, S. M. (2004). Cultural competence in hospice. American Journal of Hospice and Palliative Care, 21(1), 28-32.

Fortier, J. P., \& Bishop, D. (2004). Setting the agenda for research on cultural competence in health care: Final report. In: C. Brach (Ed.), U.S. Department of Health and Human Services, Office of Minority Health and Agency for Health Care Research and Quality. Retrieved March 20, 2005, from http://www.ahrq. gov/research/cultural.htm.

Geiger, H. J. (2001). Racial stereotyping and medicine: The need for cultural competence. Canadian Medical Association Journal, 164, 1699-1700.

Helms, J., \& Cook, D. (1999). Using race and culture in counseling and psychotherapy: Theory and process. Boston: Allyn \& Bacon.

Jibaja-Rusth, M. L., Kingery, P. M., Holcomb, J. D., Pruitt, B. E., 
\& Buckner, W. P. (1994). Development of a multicultural sensitivity scale. Journal of Health Education, 25, 350-357.

Kaiser, H. F. (1974). An index of factorial simplicity. Psychometrika, 39, 31-36.

Kottak, C. P. (2004). Anthropology: The exploration of human diversity (10th ed.). Boston: McGraw-Hill.

Leininger, M. M. (1991). Culture care diversity and universality: A theory of nursing. New York: National League for Nursing.

Long, J. A., Chang, V. W., Ibrahim, S. A., \& Asch, D. A. (2004). Update on the health disparities literature. Annals of Internal Medicine, 141, 805-812.

MacCallum, R. C., Widaman, K. F., Zhang, S., \& Hong, X. (1999). Sample size in factor analysis. Psychological Methods, 4, 84-99.

McDonald, R. P. (1999). Test theory: A unified treatment. Mahwah, NJ: Erlbaum.

Motwani, J., Hodge, J., \& Crampton, S. (1995). Managing diversity in health care industry: A conceptual model and an empirical investigation. Health Care Supervisor, 13(3), 16-23.

Pope-Davis, D. B., Eliason, M. J., \& Ottavi, T. M. (1994). Are nursing students multiculturally competent? An exploratory investigation. Journal of Nursing Education, 33, 31-33.

Reynolds, W. M. (1982). Development of reliable and valid short forms of the Marlowe-Crowne Social Desirability Scale. Journal of Clinical Psychology, 38, 119-125.

Rooda, L. A. (1993). Knowledge and attitudes of nurses toward culturally different patients: Implications for nursing education. Journal of Nursing Education, 32, 209-213.

Schim, S. M., Doorenbos, A. Z., Miller, J., \& Benkert, R. (2003). Development of a cultural competence assessment instrument. Journal of Nursing Measurement, 11, 29-40.
Smedley, B. D., Stith, A. Y., \& Nelson, A. R. (Eds.). (2003). Unequal treatment: Confronting racial and ethnic disparities in health care. Washington, DC: National Academies Press.

Smith, L. S. (1998). Cultural competence for nurses: Canonical correlation of two culture scales. Journal of Cultural Diversity, $5,120-126$.

Smith, L. S. (2001). Evaluation of an educational intervention to increase cultural competence among registered nurses. Journal of Cultural Diversity, 8, 50-63.

Sodowsky, G. R., Taffe, R. C., Gutkin, T. B., \& Wise, S. L. (1994). Development of the Multicultural Counseling Inventory (MCI): A self-report measure of multicultural competencies. Journal of Counseling Psychology, 41, 137-148.

Sue, D. W., Bernier, J. E., Durran, A., Feinberg, L., Pedersen, P., Smith, E. J., et al. (1982). Position paper: Cross-cultural counseling competencies. The Counseling Psychologist, 10, 45-52.

Tabachnick, B. G., \& Fidell, L. S. (2001). Using multivariate statistics (4th ed.). Boston: Allyn \& Bacon.

Tylor, E. B. (1958). Primitive culture: The origins of culture. New York: Torchbooks. (Original work published 1871).

U.S. Department of Health and Human Services, Centers for Disease Control and Prevention, National Center for Health Statistics. (2004). Health, United States, 2004. Retrieved March 5, 2005, from http://www.cdc.gov/nchs/hus.htm.

U.S. Department of Health and Human Services, Health Resources and Services Administration. (2001). Cultural competence works. Merrifield, VA: Author.

U.S. Department of Health and Human Services, Office of Disease Prevention and Health Promotion. (2001). Healthy people 2010. Retrieved March 23, 2005, from http://www.healthypeople. gov/Document/tableofcontents.htm\#volume1. 